BENTHAM OPEN

RESEARCH ARTICLE

\title{
Unmanned Ground Vehicle for Data Collection in Wireless Sensor Networks: Mobility-aware Sink Selection
}

\author{
Omar Aldabbas ${ }^{1}$, Abdelrahman Abuarqoub ${ }^{*}, 2$, Mohammad Hammoudeh ${ }^{3}$, Umar Raza $^{3}$ and Ahcène \\ Bounceur $^{4}$ \\ ${ }^{I}$ Faculty of Engineering Al-Balqa Applied University, Amman, Jordan, \\ ${ }^{2}$ Faculty of Information Technology, Middle East University, Amman, Jordan, \\ ${ }^{3}$ Faculty of Science \& Engineering, Manchester Metropolitan University, Manchester, UK \\ ${ }^{4}$ Computer Science Department, University of Brest (UBO), Brest, France
}

Received: February 21, 2016

Revised: May 30, 2016

Accepted: June 03, 2016

\begin{abstract}
Several recent studies have demonstrated the benefits of using the Wireless Sensor Network (WSN) technology in largescale monitoring applications, such as planetary exploration and battlefield surveillance. Sensor nodes generate continuous stream of data, which must be processed and delivered to end users in a timely manner. This is a very challenging task due to constraints in sensor node's hardware resources. Mobile Unmanned Ground Vehicles (UGV) has been put forward as a solution to increase network lifetime and to improve system's Quality of Service (QoS). UGV are mobile devices that can move closer to data sources to reduce the bridging distance to the sink. They gather and process sensory data before they transmit it over a long-range communication technology. In large-scale monitored physical environments, the deployment of multiple-UGV is essential to deliver consistent QoS across different parts of the network. However, data sink mobility causes intermittent connectivity and high reconnection overhead, which may introduce considerable data delivery delay. Consequently, frequent network reconfigurations in multiple data sink networks must be managed in an effective way. In this paper, we contribute an algorithm to allow nodes to choose between multiple available UGVs, with the primary objective of reducing the network reconfiguration and signalling overhead. This is realised by assigning each node to the mobile sink that offers the longest connectivity time. The proposed algorithm takes into account the UGV's mobility parameters, including its movement direction and velocity, to achieve longer connectivity period. Experimental results show that the proposed algorithm can reduce end-to-end delay and improve packet delivery ratio, while maintaining low sink discovery and handover overhead. When compared to its best rivals in the literature, the proposed approach improves the packet delivery ratio by up to $22 \%$, end-to-end delay by up to $28 \%$, energy consumption by up to $58 \%$, and doubles the network lifetime.
\end{abstract}

Keywords: Connection expiry time, Mobile node management, Mobile sink selection, Multiple sink, Received signal strength, Topology maintenance, Unmanned ground vehicle, Wireless sensor network.

\section{INTRODUCTION}

The main mission of Wireless Sensor Networks (WSNs) is to collect data from the environment and send it to end user's, where it is analysed to extract information about the monitored phenomena. The WSN can be applied in a wider range of application domains, e.g, planet exploration, battlefield surveillance, habitat monitoring, forest fire detection, and health care. In most applications, sensors are often deployed in high numbers to ensure full exposure of the monitored physical environment. Such large-scale networks are expected to generate enormous amount of data. Therefore, scalability is a critical design factor that needs to be considered to handle the transfer of heavy traffic under application-specific constraints. Single sink networks may suffer from congestion, increased end-to-end delays, data

\footnotetext{
* Address correspondence to this author at the Faculty of Information Technology, Middle East University, Amman, Jordan; Tel: (+962) 64790222; E-mail: AAbuarqoub@meu.edu.jo
} 
loss, and high energy consumption, amongst others. The traditional solution to overcome all the aforementioned problems is to organise nodes into multiple logical clusters. The nodes within each cluster will then be connected to a sink node dedicated for that cluster [1,2]. However, clustering introduces extra communication overhead and increases the network setup time, which reduces the network availability. Moreover, in large-scale deployments, such as planetary exploration, clustering may significantly increase data delivery end-to-end delay. This is mainly due to the multiple short-distance radio transmissions from one hop to another on the way to the final sink. An effective solution for data gathering from large-scale environments is to deploy multiple power-rich mobile sinks in the form of UGVs. Fig. (1) shows a UGV capable of collecting information that would be used in space exploration.

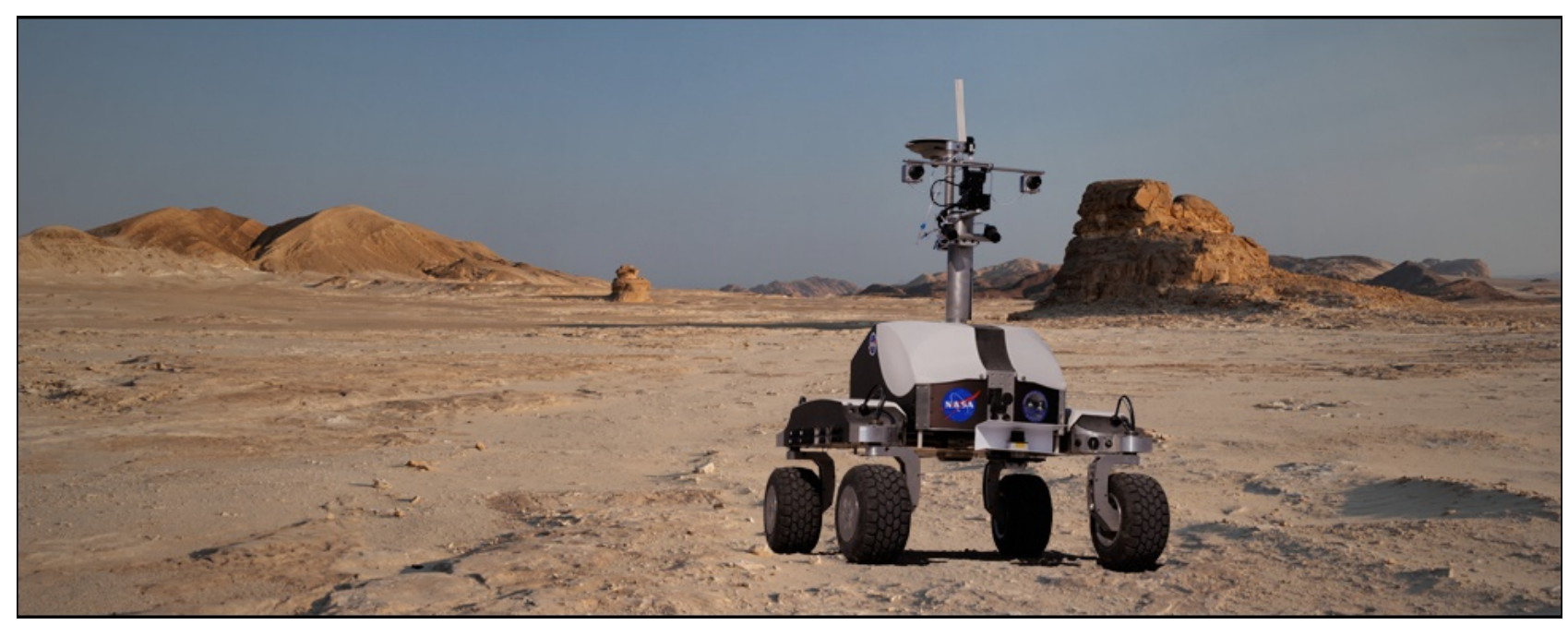

Fig. (1). Space Exploration UGV [3].

Mobile WSN is a class of WSNs that owes its name to the existence of mobile sensor nodes or sinks in the network. They are characterised by dynamic topological structures resulting from continuous link disconnections due to nodes movement outside each other's radio range. Frequent topological changes introduce new challenges, including intermittent connectivity and increased data delivery delay. Although mobility requires high management overhead, it offers significant advantages over static WSN such as: energy efficiency [4], improved sensing/radio coverage [5], enhanced information fidelity [6], greater channel capacity [7] and reliable target tracking [8].

Moving the sensor nodes towards the sink or moving the sink closer to data sources is one way to avoid the communication bottlenecks. Mobile nodes can move randomly, as in [9 - 11], on fixed trajectories, as in [12 - 15], or follow an event of interest, as in [16, 17]. Approaches such as [18 - 21], suggest moving the sink close to data sources to perform local data collection and analysis. This has also been shown to be an effective way for reducing network congestion levels and increasing network connectivity, which lead to better sensing coverage. Furthermore, moving the sink closer to sensor nodes helps conserve power by reducing the bridging distance between the node and the sink [22]. This also increases the performance of the network in terms of communication timeliness and data loss by efficiently utilising available bandwidth [23].

The rest of this paper is organised as follows: Section II examines background and related work in the area of mobile sink networks. Section III presents the proposed sink discovery and selection algorithm. It also gives the details of how the proposed algorithm handles orphaned nodes to maintain their connectivity. Section IV presents the proposed algorithm experimental evaluation results. Section V concludes the paper.

\section{RELATED WORK}

The deployment of multiple UGV for data gathering is an effective solution in environments where events of interest may simultaneously occur at several location. The availability of multiple UGV sink nodes also helps to reduce communication bottlenecks, which may lead to reduced packet loss, end-to-end delay and energy consumption. Multiple UGV sink nodes also provide the much needed redundancy in situations where one UGV is obstructed by a physical obstacle or broken due to software, hardware or even mechanical failures. Furthermore, in multiple mobile sink network, the data transmission workload is shared among all the sinks. Therefore, energy consumption is balanced and the lifetime of the networks is prolonged. 
There is a wide body of literature devoted to the study of multi-sink sensor networks. Energy efficient routing protocols for multi-sink sensor networks is investigated in [24, 25]. The placement and relocation of multi-sinks is investigated in [26]. Data collection approach to support mobility with multi-sinks is presented in [27]. However, there is only a handful set of papers directly addressing the problem of sink selection in mobile environments.

In [28], sensor nodes randomly select and join from nearby mobile sinks. Packets are routed using the shortest path from each source to its selected sink. Generally, this strategy suffers from high packet loss, as congestion may occur in several areas of the network. Congestion also increases the communication delays, due to retransmission and longer queuing times. In other approaches, such as [29, 30], nodes connect to the geographically closest mobile sink. Similarly, in the more recent approaches [31 - 33], nodes use the Received Signal Strength (RSS) to select a mobile sink. Using the RSS as the only sink selection metric may lead to unequal workload on mobile sinks. Additionally, a node could connect to the nearest mobile sink that may be moving away from it, leading to a more frequent network reconfiguration, hence, increasing the topology maintenance signalling overhead.

The above approaches dealt with sink selection without taking into consideration crucial mobility parameters such as, node movement speed and direction. The choice of a mobile sink has direct impact on the frequency of network reconfiguration, data delivery cost, communication reliability and network lifetime. Thus, joining the 'best' sink node is a very critical, but sophisticated task. Various factors need to be considered for selecting a mobile sink including the distance between the mobile sink and the selecting sensor node, link reliability, as well as the movement speed and direction of the mobile sink.

In this paper, we consider the challenge faced by sensor nodes when selecting a mobile sink among several alternatives. The proposed algorithm, called Optimal Mobile Sink Selection algorithm (OMSS), aims to improve the stability of the network and reduce the frequency of topology reconfiguration. To this end, the OMSS is based on the principle that a sensor node will be able to estimate the connection duration with a mobile sink. Using the UGV mobility parameters, i.e., speed and direction, link reliability and the relative location of the mobile sink from the sensor node, the latter can calculate a parameter called the Connection Expiration Time (CET). CET is the duration of time that a sensor node would remain reliably connected to a mobile sink before it loses connectivity due to the lack of a communication route (disconnection). The mobile sink that has the lowest CET offers a stable network for longer time.

In the following section, the details of mobile sink discover are given. This includes the details of calculating CET and how it is used by sensor nodes.

\section{MOBILE SINK DISCOVERY AND SELECTION}

Mobile sink discovery can be classified into three main categories: proactive, e.g [34], reactive, e.g [35], and hybrid, e.g [36]. In the proactive approach, the mobile data collector periodically broadcasts its presence. When an advertisement message is received by a sensor node, that node creates a route to the broadcasting data collector. This results in many duplicated messages consuming valuable bandwidth and energy. In the reactive approach, discovery messages to initialise or update connections are sent by sensor nodes. Each sensor node broadcasts a connection request message. When a data collector receives this message, it unicasts a reply containing its address. This approach saves bandwidth and energy as requests are sent only on demand. The main drawbacks of this approach is the high latency in data collector discovery and the formation of bottlenecks in areas close to data collectors. OMSS adopts a hybrid mobile sink discovery approach that combines the advantages of both the proactive and reactive approaches. The proactive approach is used by OMSS during its initialisation phase immediately after the network is deployed, and the reactive approach is used by OMSS to join orphaned nodes to the network. Orphaned sensor nodes are the nodes, which are not associated with the network hierarchy. It is possible for an orphan node to have descendants that are not orphans.

Initially, when the WSN is deployed, every mobile sink proactively discovers neighbouring nodes by broadcasting (sink_advertisement) messages containing its address, location and available resources. Since wireless communications is inherently broadcast, all advertisement messages will be eventually heard by nodes within the sender's radio range. When nodes re-broadcast the discovery message, serious message redundancy, collisions, and contention issues will occur. To reduce the impact of such problems, the scope of the mobile sink discovery message is limited to nodes that are $d$ away from the sink. The distance $d$ is defined as the maximum distance between a sensor node and the advertising mobile sink. The UGV transmission power can be adaptively adjusted to reduce or increase the number of associated nodes based on the current size of its coverage and available resources. The UGV transmission power model needs to consider the hardware design of a node and the requirements of the communication standards. The power of a certain 
signal of interest is calculated as:

$$
p=\rho S_{0} d^{-\alpha} E
$$

where $p$ is the fixed transmitter power, $S$ is the channel gain between typical Tx-Rx, $\mathrm{d}$ is the distance between source and destination, and $\alpha$ is the path-loss exponent $(\alpha>2)$. Fig. (2) shows the effective range of a mobile sink and orphan nodes.

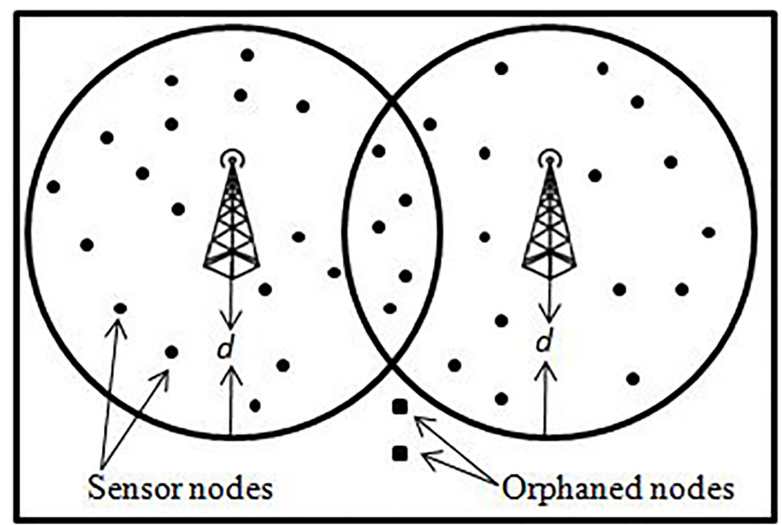

Fig. (2). Effective advertisment range of a mobile sink.

Upon receiving the Sink_advertisment message, each sensor node makes a decision about the best mobile sink to associate itself with. The decision is based on the procedure detailed in Algorithm 1, which is based on the CET. Let $n$ be a sensor node that received a Sink_advertisment message from a mobile sink. The mobile sink moves in $\theta$ direction in two-dimensional space with respect to the positive X-axis. Let $x_{n}, y_{n}$ be the location of a sensor node and $x_{\text {sink }}, y_{\text {sink }}$ be the location of the UGV. Suppose that the UGV travels at the speed of $v \mathrm{~m} / \mathrm{s}$. The velocity of the mobile sink on the $x$ and $y$ axis can be calculated using the following formula:

$$
\begin{aligned}
& v_{x}=v \times \sin \theta \\
& v_{y}=v \times \cos \theta
\end{aligned}
$$

To calculate the CET, we use the following formula that factors the location of the mobile sink, its movement speed and direction from a sensor node, link reliability and available resources.

$$
\operatorname{CET}=\frac{-\left(\mathrm{v}_{\mathrm{y}} *\left|\mathrm{x}_{\operatorname{sink}}-\mathrm{x}_{\mathrm{n}}\right|\right)+\sqrt{\left(\mathrm{v}_{\mathrm{x}}^{2}+\mathrm{v}_{\mathrm{y}}^{2}\right) \mathrm{R}^{2}-\left(\mathrm{v}_{\mathrm{y}} *\left|\mathrm{y}_{\text {sink }}-\mathrm{y}_{\mathrm{n}}\right|-\mathrm{v}_{\mathrm{x}} *\left|\mathrm{x}_{\operatorname{sink}}-\mathrm{x}_{\mathrm{n}}\right|\right)^{2}}}{\left(\mathrm{v}_{\mathrm{x}}^{2}+\mathrm{v}_{\mathrm{y}}^{2}\right)}+\mathcal{L}+\mathrm{K}
$$

where $\mathrm{K}$ is a constant of proportionality for the workload adjustment.

The link reliability $L$ is measured in terms of the weighted average of the probability $p$ of successful packet reception by a mobile sink $s$ from node $n$. Because these communication links are bidirectional, we consider the weighted average of probabilities of both transmission directions. $L$ is defined as:

$$
\mathcal{L}=\frac{1 \mathrm{P}_{\mathrm{n}, \mathrm{s}}+1 \mathrm{P}_{\mathrm{s}, \mathrm{n}}}{2}
$$

Algorithm 1 presents steps used by a sensor node to determine the optimal mobile sink to join. We define max conection as the remaining connectivity time to the current mobile sink. When a sensor node is not connected to a mobile sink, it waits for a short period to allow for advertisement from all mobile sinks in its vicinity to arrive. Then, it joins the mobile sink that offers the highest ECT value. If a sensor node, which is currently associated to a mobile sink, receives an advertisement message with a better CET value, then it leaves the current sink and joins the new one. If the node is within the vicinity of multiple mobile sinks with similar CET value, then the node joins the sink with the lowest workload level. 
Algorithm 1: Optimal mobile sink selection algorithm.

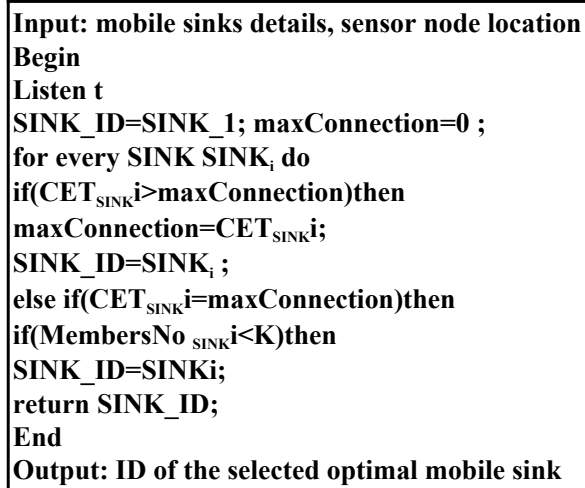

After making the decision, each sensor node replies with a (newConn) message to the chosen mobile sink. Finally, the mobile sink register nodes replies to its Sink_advertisment as connected nodes. Nodes that are not in the effective distance of any mobile sink are considered as orphaned nodes. These nodes use reactive discovery method, which we outline latter at the end of this section.

Fig. (3) illustrates the application of Algorithm 1 to a practical mobile sink selection problem. A sensor node, $n$, located at coordinates $(0,0)$ has received two Sink_advertisment messages from those two mobile sinks. Let the radio range of the mobile sinks and the sensor node be $R=100 \mathrm{~m}$. $\mathrm{SINK}_{\mathrm{A}}$ is $63 \mathrm{~m}$ far from $n$, and moving away with a direction angle of $220^{\circ}$. $\mathrm{SINK}_{\mathrm{B}}$ is $72 \mathrm{~m}$ far from $n$, and moving towards the node with a direction angle of $220^{\circ}$. Both sinks are moving with the same speed of $5 \mathrm{~m} / \mathrm{s}$. Although the distance between $n$ and $\mathrm{SINK}_{\mathrm{A}}$ is shorter than that to $\mathrm{SINK}_{\mathrm{B}}$, the CET of the $\mathrm{SINK}_{\mathrm{B}}$ is longer. This is because $\mathrm{SINK}_{\mathrm{B}}$ is moving towards the sensor node, and hence, it would stay connected to the node for 29 second. In contrast, $\mathrm{SINK}_{\mathrm{A}}$ is moving away from $n$ and will it will lose connectivity with $n$ after 29 seconds. Accordingly, the optimal sink for the sensor node $n$ is $\operatorname{SINK}_{\mathrm{B}}$, which is the sink that would remain connected for a longer time.

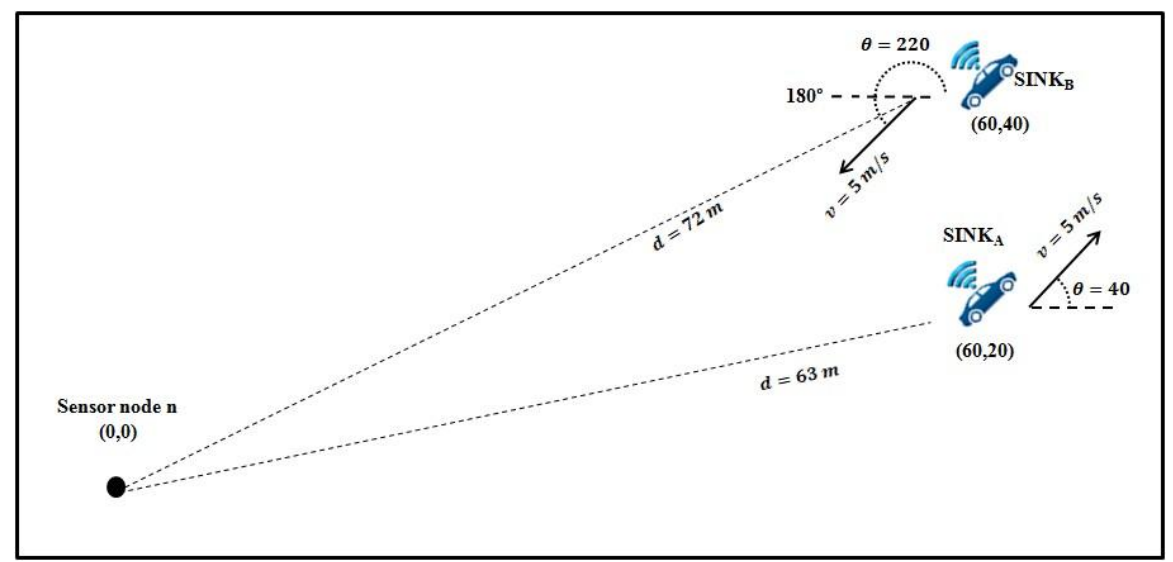

Fig. (3). Practical example to illustrate the application of Algorithm 1.

In dynamic mobile networks, orphaned node, i.e., a node that is not connected to any mobile sink is a very common problem. This could happened when the link connecting a node to its serving sink is broken, or when a node is outside the effective radio range of all sinks in the network. This leads to network segmentation problems, where the network is divided into many unconnected segments. Orphaned nodes or isolated network segments do not participate in the network as their data can not be delivered to the end user. To overcome these problems, the orphaned node reactively discover the optimal sink by following these steps:

1. If a node does not receive an advertisement from a mobile sink or gets disconnected, it waits for a back-off interval.

2. If the node still did not receive an advertisement, it sends out a Sink_solistaion message to its neighbours to 
obtain their sink node(s) information.

3. If the neighbours are also orphaned, the node enters another back off interval to allow their neighbour to obtain the sink information. Otherwise, the neighbours forward the message to the sink through multi-hop communication and wait for reply.

4. The sink sends its information Sink_reply to the forwarding node.

5. The forwarding node receives the Sink_reply message and forwards it to the orphaned node

6. Upon receiving the Sink_reply messages about the reachable sinks, the orphaned node executes Algorithm 1 to choose the optimal sink is based on the CET.

7. The orphaned node sends the optimal sink a newConn message.

8. The chosen sink waits for a backoff interval waiting for other newConn messages from other orphaned nodes.

9. The chosen sink registers the orphaned node(s) as a connected node.

Unlike the exhausted/dead nodes, orphaned nodes can still receive and transmit messages; thus, it is possible to restore them to join the network. Handling and minimising the number of orphaned nodes preserve high sensing coverage and improves the accuracy of extracted information. Thus, connecting orphaned nodes alleviates network segmentation and energy depletion problems.

\section{PERFORMANCE EVALUATION RESULTS}

This section presents the experimental results of OMSS performance against two of its rivals from the literature. In the first set of experiments, OMSS is compared against the RSS scheme. Both approaches were implemented in the NS-3 simulator. The focus of these experiments is to study the performance of OMSS compared to RSS in terms of energy consumption, end-to-end delay, Packet Delivery Ratio (PDR) and load balancing. In the second experiment, OMSS energy consumption is compared against that's of the Stateless Weight Routing with Multiple Sinks (MS-SWR) [37].

\section{A. Experiment 1}

100 sensor nodes were deployed at random in a square region of $100 \mathrm{~m} \times 100 \mathrm{~m}$. All sensor nodes have wireless radio range of 10 meters. The transmission and reception power of every sensor node is set to $0.033 \mathrm{j} / \mathrm{s}$. 50 data sources were randomly chosen to generate $2 \mathrm{pkts} / \mathrm{s}$ during the entire simulation. The packet size is 32 bytes for all types of messages except for data packets, which were set to 64 bytes. The initial energy of sensor nodes is enough to complete the simulation. $50 \%$ of sensor nodes are mobile, and their speed can reach up to $0.2 \mathrm{~m} / \mathrm{s}$. They move according to the random waypoint mobility model described in [38,39]. The number of mobile sinks is set to 5 . Their mobility speed reaches $0.5 \mathrm{~m} / \mathrm{s}$. They also move according to the random waypoint mobility model. Their maximum wireless radio range is $20 \mathrm{~m}$. A summary of the simulation parameters and their respective values is shown in Table 1. The simulation parameters values are applicable to the iMote2 [40] hardware platform.

Table 1. Simulation parameters experiment 1.

\begin{tabular}{|c|c|}
\hline Parameter & Value \\
\hline Number of nodes & $100 \mathrm{~m}$ \\
\hline Simulation area & $100 \mathrm{~m} \times 100 \mathrm{~m}$ \\
\hline Wireless Technology & Zigbee IEEE 802.15 .4 \\
\hline Wireless radio range (SN) & $10 \mathrm{~m}$ \\
\hline Wireless radio range (Sinks) & $20 \mathrm{~m}$ \\
\hline Number of source node & $20 \mathrm{~m}$ \\
\hline Source nodes data rate & $5 \mathrm{pts} / \mathrm{s}$ \\
\hline Number of mobile sinks & $0.5 \mathrm{~m} / \mathrm{s}$ \\
\hline Sink velocity & $250 \mathrm{kbps}$ \\
\hline Bandwidth & 64 bytes \\
\hline Data packet size & $0.033 \mathrm{w}$ \\
\hline TX power dissipation & $0.033 \mathrm{w}$ \\
\hline RX power dissipation & Random waypoint \\
\hline Mobility Model & \\
\hline
\end{tabular}

Fig. (4) plots the average PDR for OMSS and RSS at different network sizes. It can be observed that in all network 
densities, the OMSS algorithm has outperformed the RSS scheme by up to $18 \%$. This is due to the ability of OMSS to reduce the mobility management overhead and avoid congestion and bottlenecks. Generally, PDR decreases as the network grow in size and the amount of traffic increases. This is mainly due to the large of communication errors due to channel congestion and increased end-to-end delay (late packets are considered as lost). Moreover, the higher availability in the OMSS network due to the longer CET contributes to higher forwarding rate of data generated by nodes connected to the sink through multi-hops.

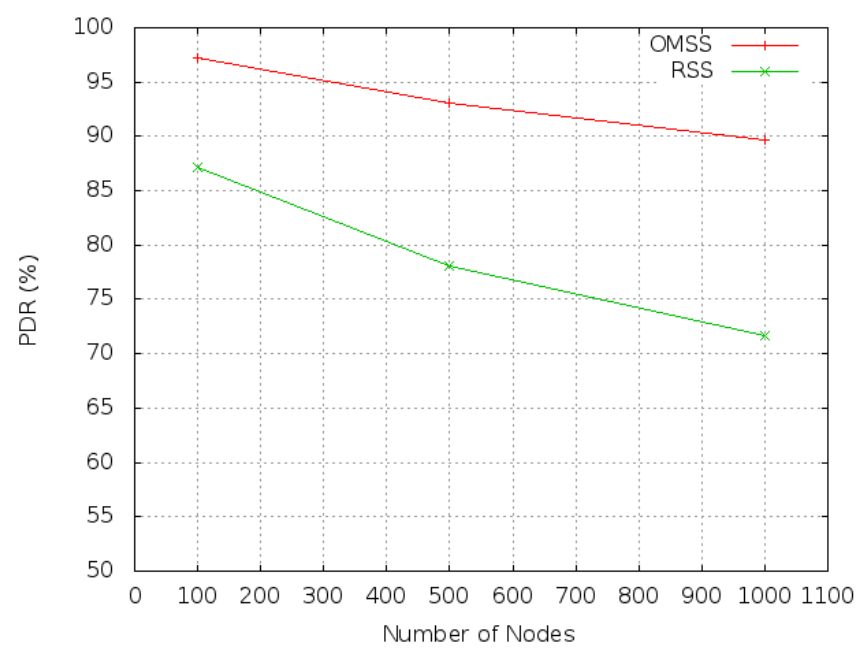

Fig. (4). PDR of OMSS against RSS.

Fig. (5) plots the average end-to-end packet communication delay in both OMSS and RSS. This delay includes the time taken by a packet to be transmitted across the network from the source to the mobile data collector. This experiment measures the variations of average end-to-end delay with respect to simulation time. The results shows that OMSS decreased the end-to-end delay by around $28 \%$, from $242 \mathrm{~m}$ to $174 \mathrm{~m}$, when compared to RSS. There are several factors contributing to this outcome. Firstly, the OMSS minimises data connectivity interruption times and maintains high network connectivity by connecting to the mobile sink that has the highest CET. This leads to stable communication links for longer periods and considerable reduction in connectivity maintenance overhead. As a result, OMSS frees more bandwidth for user data transmissions. On the other hand, RSS uses larger signalling traffic to maintain connectivity. As RSS does not consider the mobility parameters utilized by OMSS, the reconfiguration updates occur more frequently, which increases the connectivity signalling overhead. During disconnectivity periods, nodes are unable to contribute to the network by both, not sending their sensor data and forwarding other nodes traffic to the sink.

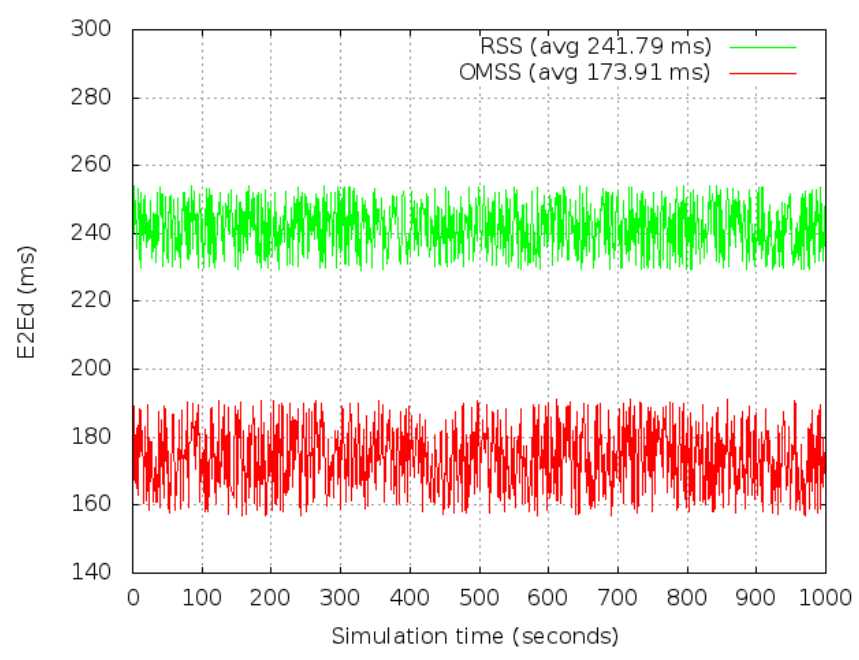

Fig. (5). End to end delay. 
Fig. (6) shows the energy consumption in both studied schemes. The graph shows that OMSS decreases energy consumption drastically when compared to RSS. The overall average energy consumption is 18.9 joles and 32.2 joules for OMSS and RSS respectively. Hence, the OMSS algorithm significantly prolongs the network lifetime by approximately $42 \%$. This is mainly due to the less frequent topological updates. Additionally, in OMSS, nodes join the mobile sink that is physically closer to them, which results in closer bridging distance between the node and its sink. Moreover, during the discovery phase, discovery flood is carefully limited to avoid unnecessary flooding of the network.

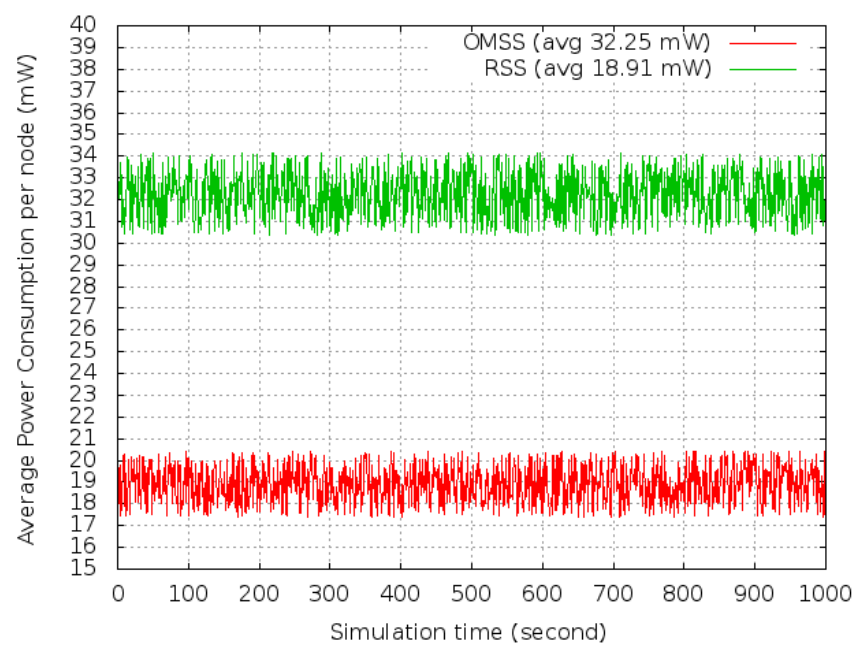

Fig. (6). Average power consumption.

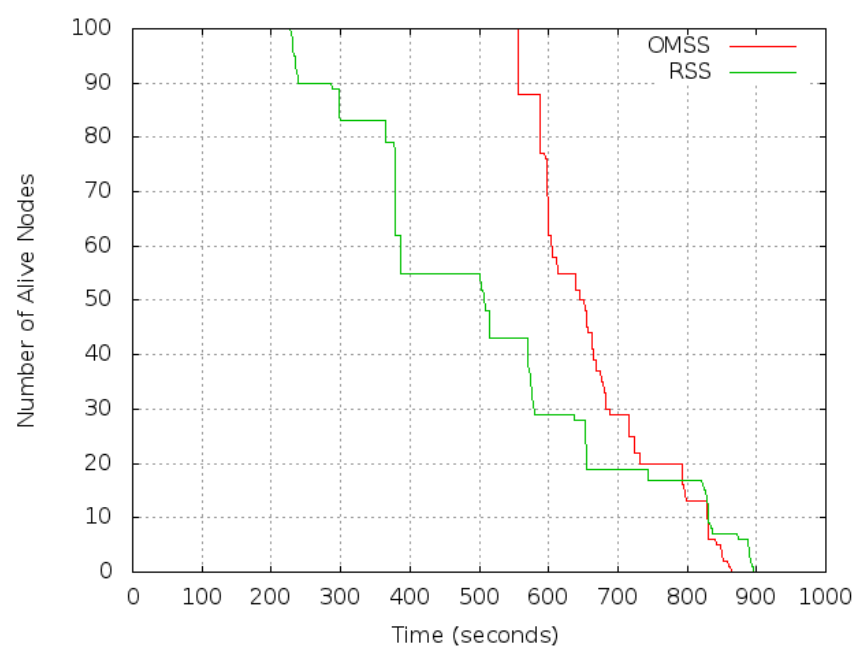

Fig. (7). Network lifetime.

The last experiment aims to measure the network lifetime when using OMSS and RSS. The experiment uses two metrics, the time for the first node to die (FND), and time the last node to die (LND). In this experiment, the initial nodes energy levels were set to 8 joules Fig. (7) shows that OMSS outperformed RSS by 58\% using the FND metric. This energy saving is mainly due to the lower amount of overhead to recover from communication interruptions and disconnections caused by nodes mobility. In RSS, nodes connect to the mobile sink that offers the strongest signal strength without considering the duration of the established link, which leads to more frequent disconnections and reconfiguration episodes. On the other hand, OMSS exploits the UGV available mobility parameters to find the most reliable link that will last longer. When the gap between FND and LND is reduced, this indicates a more balanced energy consumption among all sensor nodes in the network. In our experiment, the time between FND and LND in RSS is 660 seconds and 298 seconds in OMSS, showing 54\% improvement in energy balancing. This is partially due to thwarting network segmentation by reinstating orphaned nodes to maintain higher network connectivity. Moreover, 
OMSS reduces the number of topology reconfigurations in dense areas in particular, which help avoid unbalanced energy depletion among those nodes.

\section{B. Experiment 2}

In this simulation scenario, the system energy of OMSS is compared against the Stateless Weight Routing with Multiple Sinks (MS-SWR) [37]. The authors of MS-SWR only published the results of their energy consumption experiments. Here, the parameters for MS-SWR were obtained from the results published in [37]. A small-scale network is constructed with 50 stationary sensor nodes. Sensor nodes have wireless radio range of 10 meters. Data sources are to generate 0.05 during the entire simulation. The initial energy of sensor nodes is 1000 joul per node. The number of mobile sinks is set to 16 . Their mobility speed reaches $0.5 \mathrm{~m} / \mathrm{s}$ They also move according to the random waypoint mobility model. Their maximum wireless radio range is . A summary of the simulation parameters and their respective values are shown in Table 2.

Table 2. Simulation parameters scenario (2).

\begin{tabular}{|c|c|}
\hline Parameter & Value \\
\hline Number of nodes & 50 \\
\hline Simulation area & $100 \mathrm{~m} \times 100 \mathrm{~m}$ \\
\hline Wireless Technology & Zigbee IEEE 802.15 .4 \\
\hline Wireless radio range & $5 \mathrm{~m}$ \\
\hline Wireless sensing range & 50 \\
\hline Number of source node & $0.05 \mathrm{pkts} / \mathrm{min}$ \\
\hline Source nodes data rate & 4 \\
\hline Number of mobile sinks & Random waypoint \\
\hline Mobility Model & 1000 joules \\
\hline Initial energy (nodes) & \\
\hline
\end{tabular}

Fig. (8) shows the energy consumption in both approaches. It can be observed that OMSS scheme significantly prolongs the network lifetime. The main factor accounting for this outcome is that the OMSS scheme is able to reduce the mobility management overhead by considerably reducing the amount of communication and signalling used for sinks mobility management. However, MS-SWR uses more mobility management traffic to establish multiple paths to the sink. In contrast, in OMSS, the link reliability is considered during the sink selection process to guarantee high PDR without any extra overhead. Furthermore, MS-SWR is designed to work with mobile sinks only; when applied to multiple mobile sinks and mobile sensor nodes, the energy consumption deteriorates. Adding more sinks increases the transmissions in flooding. Moreover, MS-SWR consumes a lot of energy in beaconing.

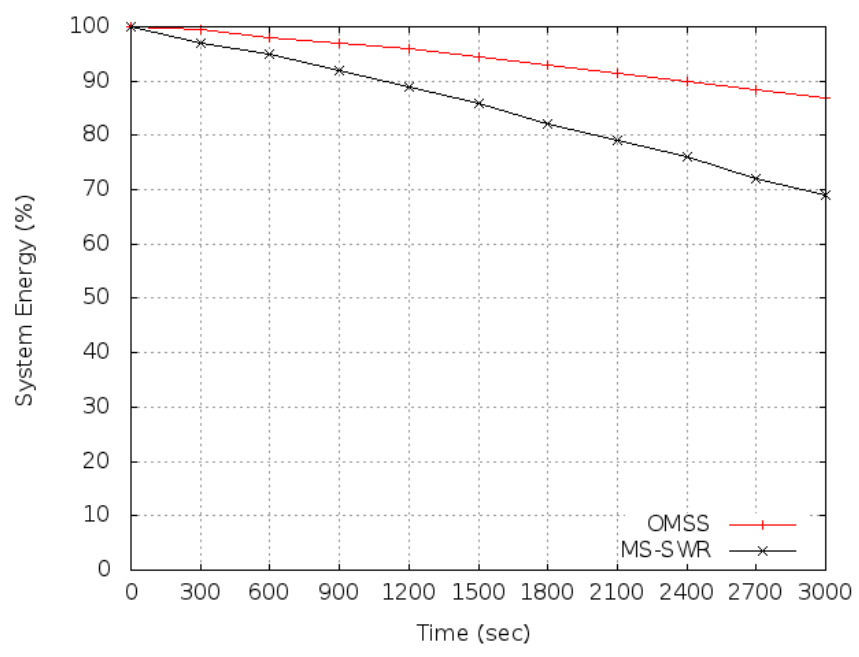

Fig. (8). System energy OMSS vs. MS-SWR. 


\section{CONCLUSION}

The utilisation of multiple UGVs as mobile data collectors is a promising solution for reliable information extraction from mobile WSNs. It allows networks operate for longer, making them financially viable technologies. To illustrate, the biggest financial cost of deploying a WSN for space exploration (e.g., [3]) is in carrying and deploying the nodes in the outer space. Besides being essential to monitor large environments, in this work we have proven that mobile nodes can double the network life while providing higher data quality in terms of end-to-end delay.

To harvest the full potential of node's mobility, we presented an algorithm to allow sensor nodes to select the mobile sink that offers the best service for the longest period. Experimental evaluation results demonstrated that the proposed algorithm outperformed two of its best rival in the literature in terms of PDR, end-to-end delay, energy consumption, and sink discovery overhead.

In the future, we plan to study the performance of the proposed OMSS algorithm under different mobility models using a modern simulation tool such as CupCarbon [41]. Additionally, more evaluation parameters have to be measured, such as, mobility management cost, sink discovery cost, and orphaned nodes restoration time. Finally, an interesting future line work is exploiting the benefits of the proposed solution in other areas of ad-hoc wireless networks, such as vehicular networks.

\section{CONFLICT OF INTEREST}

The authors confirm that this article content has no conflict of interest.

\section{ACKNOWLEDGEMENTS}

Declared none.

\section{REFERENCES}

[1] M. Hammoudeh, and R. Newman, "Adaptive routing in wireless sensor networks: QoS optimisation for enhanced application performance", Inf. Fusion, vol. 22, pp. 3-15, 2013.

[http://dx.doi.org/10.1016/j.inffus.2013.02.005]

[2] M. Hammoudeh, and R. Newman, "Information extraction from sensor networks using the Watershed transform algorithm", Inf. Fusion, vol. 22, pp. 39-49, 2013. [http://dx.doi.org/10.1016/j.inffus.2013.07.001]

[3] Unmanned Robotic Vehicle. Available from: http://www.novatel.com/technology-in-action/mars-rovers/\#rover-testing. 8 July 2015

[4] S.A. Munir, B. Ren, W. Jiao, B. Wang, D. Xie, and J. Ma, "Mobile wireless sensor network: architecture and enabling technologies for ubiquitous computing", In: 21 International Conference on Advanced Information Networking and Applications Workshops, 2007 (AINAW'07), 2007, pp. 113-120

[http://dx.doi.org/10.1109/AINAW.2007.257]

[5] V. Coskun, "Relocating sensor nodes to maximize cumulative connected coverage in wireless sensor networks", Sensors (Basel Switzerland), vol. 8 , pp. 2792-2817, 2008 .

[http://dx.doi.org/10.3390/s8042792]

[6] C. Zhu, L. Shu, T. Hara, L. Wang, and S. Nishio, "Research issues on mobile sensor networks", In: $5^{\text {th }}$ International ICST Conference on Communications and Networking in China (CHINACOM), 2010, pp. 1-6.

[7] M. Grossglauser, and D.N. Tse, "Mobility increases the capacity of ad hoc wireless networks", IEEE/ACM Trans. Netw., vol. 10, pp. 477-486, 2002 .

[http://dx.doi.org/10.1109/TNET.2002.801403]

[8] S. Sivaramakrishnan, and A. Al-Anbuky, "Analysis of network connectivity: wildlife and sensor network", In: Telecommun. Netw. Appl. Conf. (ATNAC), 2009, pp. 1-6. [http://dx.doi.org/10.1109/ATNAC.2009.5464822]

[9] S. Jain, R.C. Shah, W. Brunette, G. Borriello, and S. Roy, "Exploiting mobility for energy efficient data collection in wireless sensor networks", Mob. Netw. Appl., vol. 11, no. 3, pp. 327-339, 2006. [http://dx.doi.org/10.1007/s11036-006-5186-9]

[10] R.C. Shah, S. Roy, S. Jain, and W. Brunette, "Data MULEs: modeling a three-tier architecture for sparse sensor networks", In: Proceedings of the $1^{\text {st }}$ IEEE 2003 International Workshop on Sensor Network Protocols and Applications, 2003, pp. 30-41. [http://dx.doi.org/10.1109/SNPA.2003.1203354]

[11] D.N. Jayasingha, "Wireless Sensor Network Testbed for Mobile Data Communication", In: $4^{\text {th }}$ International Conference on Information and Automation for Sustainability, 2008 (ICIAFS 2008), 2008, pp. 97-103. [http://dx.doi.org/10.1109/ICIAFS.2008.4783994] 
[12] G. Anastasi, M. Conti, and M. Di Francesco, "Data collection in sensor networks with data mules: An integrated simulation analysis", In: IEEE Symposium on Computers and Communications, 2008 (ISCC 2008), pp. 1096-1102. [http://dx.doi.org/10.1109/ISCC.2008.4625629]

[13] A. Chakrabarti, A. Sabharwal, and B. Aazhang, "Communication power optimization in a sensor network with a path-constrained mobile observer", ACM Trans. Sens. Netw., vol. 2, pp. 297-324, 2006. [http://dx.doi.org/10.1145/1167935.1167936]

[14] W. Alsalih, "Routing to a mobile data collector on a predefined trajectory", In: IEEE International Conference on Communications (ICC'09), 2009, pp. 1-5.

[http://dx.doi.org/10.1109/ICC.2009.5199428]

[15] D. Jea, A. Somasundara, and M. Srivastava, "Multiple controlled mobile elements (data mules) for data collection in sensor networks", Proceedings of the $1^{\text {st }}$ IEEE International Conference on Distributed Computing in Sensor Systems (DCOSS'05), 2005, pp. $244-257$. [http://dx.doi.org/10.1007/11502593_20]

[16] M. Xi, "Run to potential: sweep coverage in wireless sensor networks", In: International Conference on Parallel Processing (ICPP'09), 2009, pp. 50-57.

[http://dx.doi.org/10.1109/ICPP.2009.43]

[17] L. He, Y. Zhuang, J. Pan, and J. Xu, "Evaluating on-demand data collection with mobile elements in wireless sensor networks", In: IEEE 72 ${ }^{\text {nd }}$ Vehicular Technology Conference Fall (VTC 2010-Fall), 2010, pp. 1-5. [http://dx.doi.org/10.1109/VETECF.2010.5594515]

[18] G. Pantziou, "Mobile sinks for information retrieval from cluster-based WSN islands", In: Proceedings of the $8^{\text {th }}$ International Conference on Ad-Hoc, Mobile and Wireless Networks, 2009, pp. 213-226. [http://dx.doi.org/10.1007/978-3-642-04383-3_16]

[19] H. Lee, M. Wicke, B. Kusy, O. Gnawali, and L. Guibas, "Data stashing: energy-efficient information delivery to mobile sinks through trajectory prediction", In: Proceedings of the $9^{\text {th }}$ ACM/IEEE International Conference on Information Processing in Sensor Networks (IPSN'10), 2010, pp. 291-302.

[http://dx.doi.org/10.1145/1791212.1791247]

[20] L. Tzevelekas, and I. Stavrakakis, "Sink mobility schemes for data extraction in large scale WSNs under single or zero hop data forwarding", In: European Wireless Conference (EW), 2010, pp. 896-902.

[http://dx.doi.org/10.1109/EW.2010.5483528]

[21] H. Sabbineni, and K. Chakrabarty, "Datacollection in event-driven wireless sensor networks with mobile sinks", Int. J. Distrib. Sens. Netw., vol. 2010, pp. 1-12, 2010 .

[22] A. Kinalis, S. Nikoletseas, D. Patroumpa, and J. Rolim, "Biased sink mobility with adaptive stop times for low latency data collection in sensor networks", In: IEEE Global Telecommunications Conference (GLOBECOM 2009), 2009, pp. 1-6. [http://dx.doi.org/10.1109/GLOCOM.2009.5425600]

[23] A. Kansal, "Controlled mobility for sustainable wireless sensor networks", In: $1^{\text {st }}$ Annual IEEE Communications Society Conference on Sensor and Ad Hoc Communications and Networks, IEEE (SECON 2004), 2004, pp. 1-6. [http://dx.doi.org/10.1109/SAHCN.2004.1381896]

[24] M. Koç, and I. Korpeoglu, "Controlled sink mobility algorithms for wireless sensor networks", Int. J. Distrib. Sens. Netw., vol. 2014, pp. 1-12, 2014.

[http://dx.doi.org/10.1155/2014/167508]

[25] S. Yu, B. Zhang, C. Li, and H.T. Mouftah, "Routing protocols for wireless sensor networks with mobile sinks: a survey", IEEE Commun. Mag., vol. 52, no. 7, pp. 150-157, 2014. [http://dx.doi.org/10.1109/MCOM.2014.6852097]

[26] D. Das, Z. Rehena, S. Roy, and N. Mukherjee, "Multiple-sink placement strategies in wireless sensor networks", In: $5^{\text {th }}$ International Conference on Communication Systems and Networks (COMSNETS 2013), 2013, pp. 1-7. [http://dx.doi.org/10.1109/COMSNETS.2013.6465578]

[27] B. Sudhakar, and K. Sangeetha, "Multi sink based data collection scheme for wireless sensor networks", Int. J. Innovative Res. Comp. Commun. Eng., vol. 2, pp. 1139-1146, 2014.

[28] I. Shukla, and N. Meghanathan, "Impact of leader selection strategies on the PEGASIS data gathering protocol for wireless sensor networks", Ubiquitous Comput. Commun. J., vol. 4, pp. 20-29, 2005.

[29] D. Luo, D. Zuo, and X. Yang, "An optimal sink selection scheme for multi-sink wireless sensor networks", In: International Conference on Computer Science and Information Technology (ICCSIT' 08), 2008.

[http://dx.doi.org/10.1109/ICCSIT.2008.161]

[30] D. Gao, H. Qian, Z. Wang, and J. Chen, "Study and design an anycast routing protocol for wireless sensor networks", J. Netw, vol. 6, no. 12, pp. 1726-1733, 2011. [http://dx.doi.org/10.4304/jnw.6.12.1726-1733]

[31] R. Paulus, G. Singh, and R. Tripathi, "Energy efficient data transmission through relay nodes in wireless sensor networks", $A C E E E$ International Journal on Network Security 3, vol. 3, no. 1, pp. 40-45, 2012 . 
[32] D. Jose, and G. Sadashivappa, "Mobile sink assisted energy efficient routing algorithm for wireless sensor networks", World Comp. Sci. Inform. Technol. J., vol. 5, pp. 16-22, 2015. [WCSIT].

[33] A.I. Alhasanat, K.D. Matrouk, H.A. Alasha'ary, and Z.A. Al-Qadi, "Connectivity-based data gathering with path-constrained mobile sink in wireless sensor networks", Wireless Sensor Netw., vol. 6, no. 6, pp. 118-128, 2014.

[http://dx.doi.org/10.4236/wsn.2014.66013]

[34] A. Shahid, M.Z. Hameed, S.A. Khan, and Z.A. Khan, "Proactive multipath data dissemination for multimedia sensor networks", In: $15^{\text {th }}$ International Multitopic Conference (INMIC), 2012. [http://dx.doi.org/10.1109/INMIC.2012.6511476]

[35] J. Niu, L. Cheng, Y. Gu, L. Shu, and S.K. Das, "R3E: reliable reactive routing enhancement for wireless sensor networks", IEEE Trans. Industr. Inform., vol. 10, pp. 784-794, 2014.

[http://dx.doi.org/10.1109/TII.2013.2261082]

[36] C. Hsung-Pin, and H. Shun-Chih, "A hybrid intelligent protocol in sink-oriented wireless sensor networks", In: International Conference on Information Security and Intelligence Control (ISIC 2012), 2012, pp. 57-60.

[37] M. Soyturk, and T. Altilar, "A routing algorithm for mobile multiple sinks in large-scale wireless sensor networks", In: $2^{\text {nd }}$ International Symposium on Wireless Pervasive Computing, 2007.. [http://dx.doi.org/10.1109/ISWPC.2007.342575]

[38] J. Broch, D.A. Maltz, D.B. Johnson, Y.C. Hu, and J. Jetcheva, "A performance comparison of multi-hop wireless ad hoc network routing protocols", In: Proceedings of the $4^{\text {th }}$ Annual ACM/IEEE International Conference on Mobile Computing and Networking, 1998, Dallas, Texas, USA.

[http://dx.doi.org/10.1145/288235.288256]

[39] D. Johnson, and D. Maltz, "Dynamic source routing in ad hoc wireless networks", In: T. Imielinski, and H. Korth, Eds., Mobile Computing, vol. 353. Springer US, 1996, pp. 153-181. [http://dx.doi.org/10.1007/978-0-585-29603-6_5]

[40] "Intel, "Intel Mote 2", In: Engineering Platform Data Sheet ed., 2006

[41] A. Bounceur, "CupCarbon: a new platform for designing and simulating smart-city and IoT wireless sensor networks (SCI-WSN)", In: Proceedings of the International Conference on Internet of Things and Cloud Computing, 2016, Cambridge, United Kingdom. [http://dx.doi.org/10.1145/2896387.2900336]

(C) Aldabbas et al.; Licensee Bentham Science Publishers

This is an open access article licensed under the terms of the Creative Commons Attribution-Non-Commercial 4.0 International Public License (CC BY-NC 4.0) (https://creativecommons.org/licenses/by-nc/4.0/legalcode), which permits unrestricted, non-commercial use, distribution and reproduction in any medium, provided the work is properly cited. 